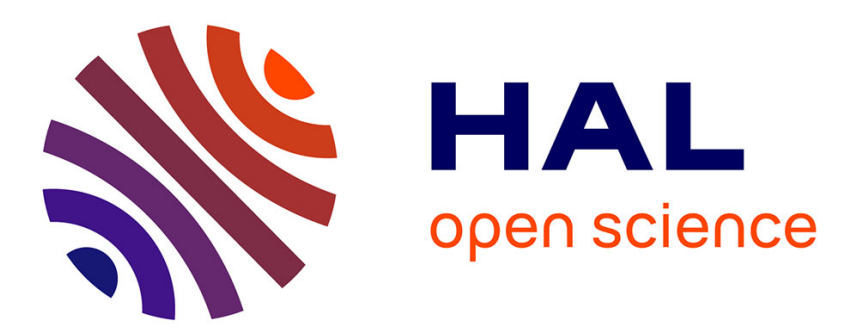

\title{
Discussion of the application of finite Volterra series for the modeling of the oscillation behavior of ultrasound contrast agents
}

\author{
Michal Mleczko, Michiel Postema, Georg Schmitz
}

\section{- To cite this version:}

Michal Mleczko, Michiel Postema, Georg Schmitz. Discussion of the application of finite Volterra series for the modeling of the oscillation behavior of ultrasound contrast agents. Applied Acoustics, 2009, 70 (10), pp.1363-1369. 10.1016/j.apacoust.2008.09.012 . hal-03193309

\author{
HAL Id: hal-03193309 \\ https://hal.science/hal-03193309
}

Submitted on 15 Apr 2021

HAL is a multi-disciplinary open access archive for the deposit and dissemination of scientific research documents, whether they are published or not. The documents may come from teaching and research institutions in France or abroad, or from public or private research centers.
L'archive ouverte pluridisciplinaire HAL, est destinée au dépôt et à la diffusion de documents scientifiques de niveau recherche, publiés ou non, émanant des établissements d'enseignement et de recherche français ou étrangers, des laboratoires publics ou privés. 


\title{
Discussion of the application of finite Volterra series for the modeling of the oscillation behaviour of ultrasound contrast agents
}

\author{
Michał Mleczko* \\ Institute of Medical Engineering, Ruhr-Universität Bochum, Universitätsstr. 150, 44780 Bochum, Germany \\ Michiel Postema \\ Department of Engineering, University of Hull, Kingston upon Hull, HU6 7RX, Great Britain \\ Georg Schmitz \\ Institute of Medical Engineering, Ruhr-Universität Bochum, Universitätsstr. 150, 44780 Bochum, Germany
}

\begin{abstract}
Ultrasound contrast agents consist of microbubbles with diameters in the micrometer range. Excited by ultrasound, these bubbles exhibit highly nonlinear oscillation. This paper evaluates the usage of Volterra series for the modeling of the oscillation behavior of contrast agent microbubbles. Feasibility is determined by implementation and application of an identification algorithm to oscillation data obtained from numerical simulations of a free gas bubble with a resting radius $r_{0}=1 \mu \mathrm{m}$. For insonification pressures up to $100 \mathrm{kPa}$, a cubic model allowed for a mean-square error of less than $-16 \mathrm{~dB}$ with respect to the reference signal. Analysis of the response to narrowband signals showed that the achievable mean-square error is further reduced for the bandwidth available to typical ultrasound transducers used for clinical diagnostics.
\end{abstract}

Key words: ultrasound contrast agent, microbubble, Volterra series, Rayleigh-Plesset, system identification, nonlinear oscillation PACS:

\section{Introduction}

Ultrasound contrast agents consist of gas filled microbubbles with diameters ranging from $1-10 \mu \mathrm{m}$. Since the acoustic impedance of these gas cavities differs significantly from the surrounding liquid and these bubbles are resonant at frequencies used for diagnostic imaging, microbubbles are strong scatterers. This enables the quantification of perfusion in small capillaries. The oscillation of the microbubble is of highly nonlinear nature $[1,2]$, the contrast between the contrast agent and the surrounding tissue may thus be increased by isolating signal components generated by nonlinear systems. UCA detection modalities used in commercial ultrasound systems $[3,4]$ enable contrast agent imaging by comparison of responses to insonification pulses with different amplitudes and polarities. Sound propagation in

\footnotetext{
* Corresponding author

Email: michal.mleczko@rub.de

Tel: $+49-234-3227659$

Fax: +49-234-3214872
}

tissue, however, may only be assumed to be linear for low acoustic pressures and small penetration depths. In medical ultrasound imaging, the nonlinearity of sound propagation may not be ignored. The attainable contrast-to-tissue ratio is thus degraded.

To increase the specifity of contrast agent detection, knowledge about microbubble oscillation is necessary. A model is thus needed which enables the description of the relevant aspects of microbubble oscillation. Starting with the development of equations for free gas bubbles [5], greybox models, based on insight into the physical background of microbubble oscillation were developed. To account for the influence of the shell of an encapsulated microbubble, these models were extended $[6,7]$.

In contrast to the process of understanding nonlinear oscillation behavior, however, a different description form may prove helpful for the development of new detection methods. In linear system theory, this is accomplished by considering the transfer function or impulse response. Nonlinear system theory also provides well-researched models which can be applied to the modeling of the oscillation of 
ultrasound contrast agents. Thus, the aim of the work presented in this paper is to evaluate a black-box nonlinear model with respect to the nonlinear oscillation of microbubbles. The choice for a suitable model was made in favor of Volterra series. These are characterized by a high versatility, enabling the description of a variety of static and dynamic nonlinear systems. Furthermore, the parameters of a Volterra series may be determined using numerically stable linear estimators.

In this paper, the application of Volterra series to microbubble oscillation modeling will be presented. Volterra series will be introduced and an identification algorithm which allows for a numerically stable estimation of the parameters of a Volterra series will be presented. The feasibility of Volterra series modeling of oscillating microbubbles will be evaluated by determination of the nonlinear components for a microbubble simulation, implemented by a modified Rayleigh-Plesset equation. The quality of the fit achieved by the Volterra series will be compared to the Rayleigh-Plesset model to evaluate the feasibility of microbubble oscillation modeling with Volterra series.

\section{Volterra series}

\subsection{Definition}

Volterra series were introduced in [8] as a Taylor series with memory. In discrete time $n$, for a series length of $N+$ 1 , the system output $y(n)$ is characterized by the series expansion

$$
\begin{aligned}
y(n) & =h_{0}+\sum_{k_{1}=0}^{N} h_{1}\left(k_{1}\right) x\left(n-k_{1}\right) \\
& +\sum_{k_{1}=0}^{N} \sum_{k_{2}=0}^{N} h_{2}\left(k_{1}, k_{2}\right) x\left(n-k_{1}\right) x\left(n-k_{2}\right) \\
& +\sum_{k_{1}=0}^{N} \sum_{k_{2}=0}^{N} \sum_{k_{3}=0}^{N} h_{3}\left(k_{1}, k_{2}, k_{3}\right) \\
& +\ldots\left(n-k_{1}\right) x\left(n-k_{2}\right) x\left(n-k_{3}\right) \\
&
\end{aligned}
$$

with $x(n)$ denoting the system input and $h_{k}\left(n_{1}, \ldots, n_{k}\right)$ denoting the $k$-dimensional impulse responses, or kernels. The system output consists of a constant component, determined by the constant kernel $h_{0}$, a linear component, implemented by a linear convolution of system input and linear impulse response, and the nonlinear components which are implemented by $k$-dimensional convolutions of the respective kernels with the $k$-th order input $\prod_{i=0}^{k-1} x\left(n-k_{i}\right)$.

\subsection{Identification of parameters}

The coefficients of the Volterra series can be identified by methods introduced in $[9,10]$. For the work conducted in this paper, the method proposed in [10] was chosen, as it features superior numerical stability. Furthermore, no assumption as to the structure of the excitation signal is needed, as long as the input signal is sufficiently rich in the sense that it is persistently exciting with respect to the system being identified [11].

Table 1

Corresponding monomials $q_{k}(n)$ and parameters $a_{k}$ when rearranging the Volterra series as introduced in (1) into the series expansion shown in Equation (2).

$$
\begin{array}{ll}
q_{0}(n)=1 & a_{0}=h_{0} \\
q_{1}(n)=x(n) & a_{1}=h_{1}(0) \\
q_{2}(n)=x(n-1) & a_{2}=h_{1}(1) \\
& \vdots \\
q_{N+2}(n)=x(n-N) & a_{N+2}=h_{1}(N) \\
q_{N+3}(n)=x(n) \cdot x(n) & a_{N+3}=h_{2}(0,0) \\
q_{N+4}(n)=x(n) \cdot x(n-1) & a_{N+4}=h_{2}(0,1)
\end{array}
$$

To determine the coefficients of the Volterra series, the system output, as given in (1) is rewritten as

$$
y(n)=\sum_{k=0}^{M-1} a_{k} q_{k}(n) .
$$

In this notation form, the kernels $h_{i}\left(k_{1}, \ldots, k_{i}\right)$ are arranged into the parameters $a_{k}$ and the input is rearranged as a list of monomials $q_{k}(n)$. Table 1 displays the rearrangement of the Volterra series into the parameters $a_{k}$ and input monomials $q_{k}(n)$. For a series length of $N+1$ samples, the total number of unique coefficients can be calculated to be

$$
M=\sum_{i=0}^{N}\left(\begin{array}{c}
N+i \\
i
\end{array}\right)
$$

In matrix form, Equation (2) may be written as

$$
\mathbf{y}=\mathbf{X a} \text {. }
$$

The output vector $\mathbf{y}$ consists of the system output

$$
\mathbf{y}=(y(0) y(1) \cdots)^{\mathrm{T}},
$$

with the input matrix $\mathbf{X}$ being defined as:

$$
\mathbf{X}=\left(\begin{array}{cccc}
q_{0}(0) & q_{1}(0) & \cdots & q_{M-1}(0) \\
q_{0}(1) & q_{1}(1) & \cdots & q_{M-1}(1) \\
\vdots & \vdots & \ddots & \vdots
\end{array}\right)
$$

while the coefficient vector a contains all kernel coefficients:

$$
\mathbf{a}=\left(\begin{array}{lll}
a_{0} & a_{1} & \cdots
\end{array}\right)^{\mathrm{T}}
$$

This equation may be solved for the parameter vector $\mathbf{a}$ by linear least-squares estimation:

$$
\mathbf{a}=\left(\mathbf{X}^{\mathrm{T}} \mathbf{X}\right)^{-1} \mathbf{X}^{\mathrm{T}} \mathbf{y}
$$


To obtain a numerically stable estimate of $\mathbf{a},(2)$ has to be rewritten as:

$$
\mathbf{X}^{\mathrm{T}} \mathbf{y}=\mathbf{X}^{\mathrm{T}} \mathbf{X} \mathbf{a}
$$

The matrix $\mathbf{X}^{\mathrm{T}} \mathbf{X}$ is positive-semidefinite, thus a Cholesky decomposition of $\mathbf{X}^{\mathrm{T}} \mathbf{X}$ may be conducted to yield a triangular matrix $\mathbf{D}$ such that

$$
\mathbf{X}^{\mathrm{T}} \mathbf{X}=\mathbf{D}^{\mathrm{T}} \mathbf{D}
$$

The solution for the coefficients $\mathbf{a}$ is conducted in two steps. Initially, an intermediate set of coefficients $\mathbf{g}$ is determined by solving

$$
\mathbf{D g}=\mathbf{X}^{\mathrm{T}} \mathbf{y}
$$

This can be conducted efficiently since $\mathbf{D}$ is of upper triangular form and the solution can be obtained by backward substitution. Subsequently, the final solution can be obtained by solving

$$
\mathbf{D}^{\mathrm{T}} \mathbf{a}=\mathbf{g}
$$

for a. As before, the triangular form of $\mathbf{D}^{\mathrm{T}}$ allows for an efficient calculation by forward substitution.

\section{Determination of the Volterra kernels of simulated microbubbles}

To prove the feasibility of the system identification approach for contrast agent microbubbles, data obtained from a microbubble-simulation was subjected to the system identification procedure outlined in Section 2. As system to be identified, a free gas bubble with a resting radius $r_{0}=1 \mu \mathrm{m}$ was chosen. The motivation for such a test system was that a free microbubble with such a diameter has a resonance frequency which is in the range of diagnostic imaging. Furthermore, an encapsulating shell was not considered to allow for the maximum amount of nonlinear oscillation which would otherwise be damped by the presence of a shell.

The simulation of contrast agent behavior was implemented by numerical solution of a modified RayleighPlesset equation. The bubble radius $r$ is given by the differential equation [12]:

$$
\begin{aligned}
\rho r \ddot{r}+\frac{3}{2} \dot{r}^{2}= & \left(p_{0}+\frac{2 \sigma}{r_{0}}\right)+\left(\frac{r_{0}}{r}\right)^{3 \Gamma}-\frac{4 \mu \dot{r}}{r} \\
& -\frac{2 \sigma}{r}-\omega^{2} \rho r^{2} \frac{\dot{r}}{c}-p_{0}+p_{a}(t)
\end{aligned}
$$

with parameters and their respective values being described in Table 2. The pressure $p_{a}(t)$ describes the incident excitation pressure.

For the excitation pressure, a discrete-time normally distributed white-noise process is used. The radius is computed using an explicit Runge-Kutta $(4,5)$ initial value solver (The Mathworks, Natick, MA). The initial values are chosen for a resting bubble with the initial radius $r_{0}$. The resulting scattered pressure is then determined by taking into account the pressure field by a radiating point source [13]. As shown in Figure 1, the computed pressure, along
Table 2

Parameters and values used for simulations of the microbubble movement as calculated from Equation (13).

$$
\begin{array}{lr}
r_{0}=1 \mu \mathrm{m} & \text { resting radius } \\
\Gamma=1.7 & \text { polytropic exponent } \\
c=1480 \mathrm{~ms}^{-1} & \text { speed of sound } \\
\mu=10^{-3} \mathrm{Pas}^{-3} & \text { shear viscosity of surrounding medium } \\
\rho=998 \mathrm{~kg} \mathrm{~m}^{-3} & \text { density of surrounding medium } \\
\sigma=0.072 \mathrm{Nm}^{-1} & \text { surface tension } \\
p_{0}=1.013 \cdot 10^{5} \mathrm{~Pa} & \text { ambient pressure }
\end{array}
$$

with the input is used to identify the kernels of a Volterra series using an implementation of the algorithm outlined before.

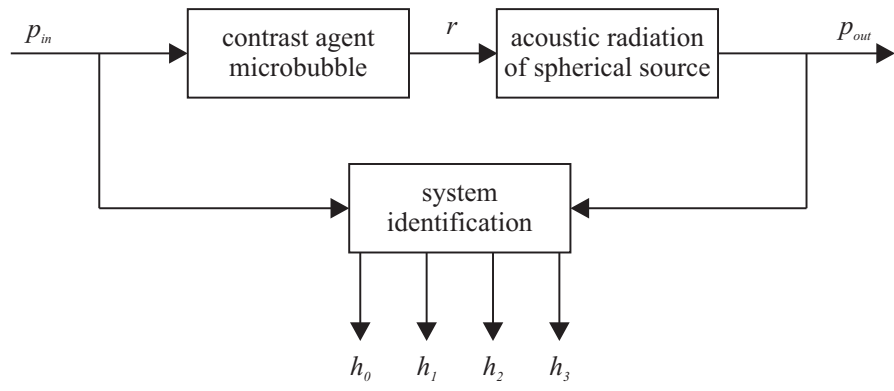

Fig. 1. Schematic of identification scheme.

\section{Results and Discussion}

\subsection{Obtained kernels}

The kernels obtained from an identification for a standard deviation of the input white noise of $100 \mathrm{kPa}$ are shown in Figures 2-4. Identification was conducted for a third order model with a maximum series length of 30 samples at a sampling frequency $f_{s}=40 \mathrm{MHz}$. The linear kernel, as shown in Figure 2, as anticipated, exhibits the typical behavior of a damped oscillator. It is characterized by a resonance frequency $f_{r}=4.6 \mathrm{MHz}$ and a fractional bandwith of $B=39 \%$. Similar oscillator characteristics can be seen in the quadratic and cubic kernels, as shown in Figures 3 and 4 .

\subsection{MSE vs. filter length and amplitude}

An important step necessary for the development of new detection methods is the determination of the correct model order and length. This enables a sufficiently accurate determination of bubble movement, without being excessively complex. For oscillating microbubbles, the identified model was reviewed by determining the mean-square error according to:

$$
\mathrm{MSE}=\frac{\left\|y_{\text {Volterra }}-y_{\mathrm{RPNNP}}\right\|_{2}}{\left\|y_{\mathrm{RPNNP}}\right\|_{2}}
$$




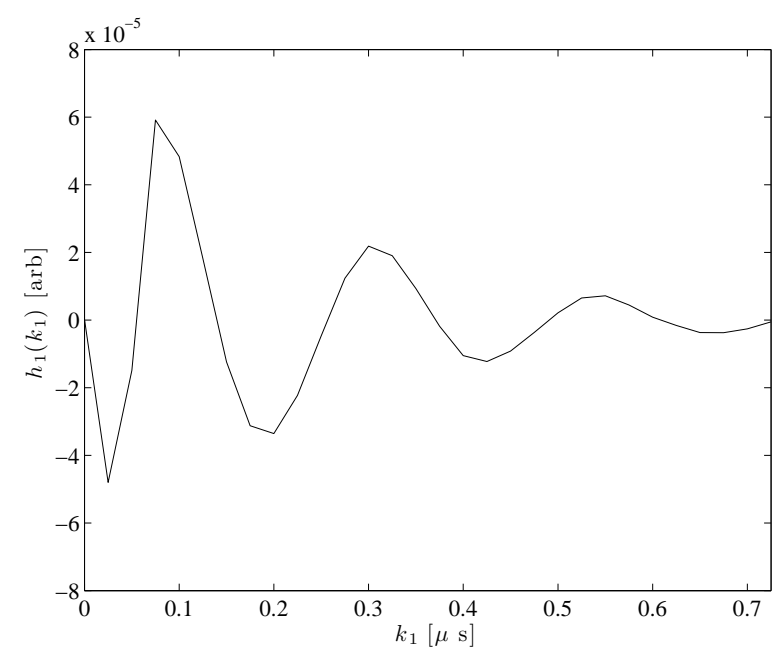

Fig. 2. Linear kernel $h_{1}$ of the simulated microbubble.

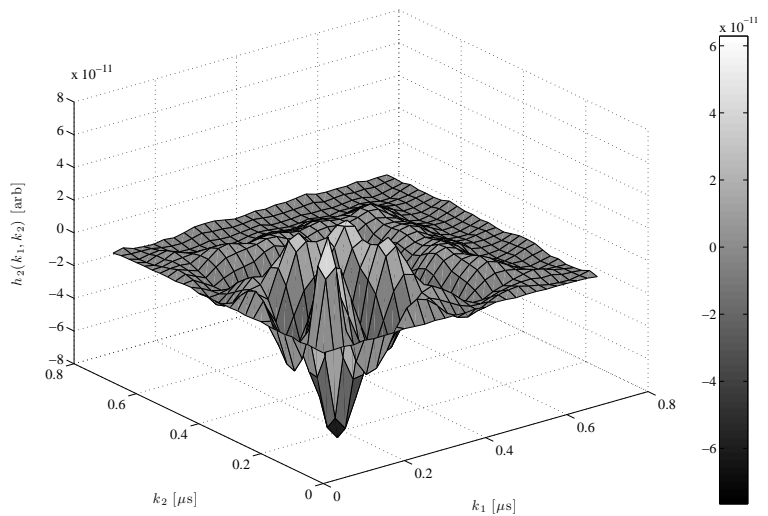

Fig. 3. Quadratic kernel $h_{2}$ of the simulated microbubble.

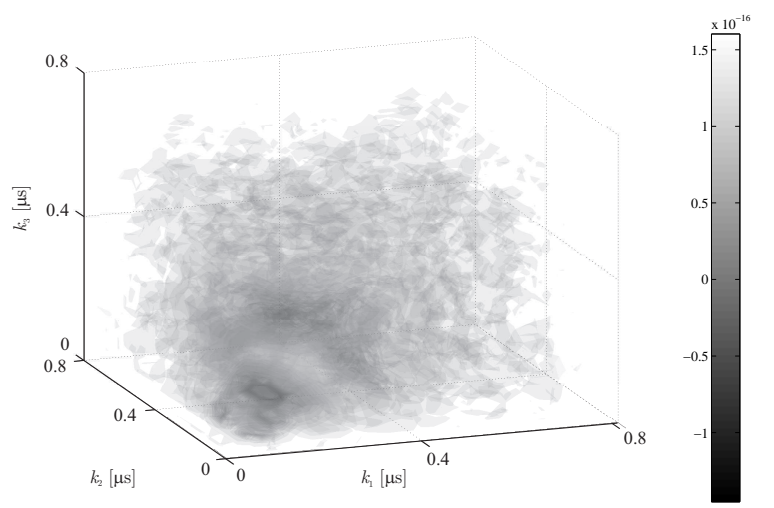

Fig. 4. Cubic kernel $h_{3}$ of the simulated microbubble.

with $y_{\text {Volterra }}$ denoting the output for the Volterra series model and $y_{\text {RPNNP }}$ denoting the output obtained from a numerical integration of the RPNNP equation. The operator $\|\cdot\|_{2}$ denotes the $l_{2}$ norm of the expression. This was conducted for different excitation amplitudes ranging from $1 \mathrm{kPa}$ to $100 \mathrm{kPa}$. Furthermore, the identified series length was varied from 2 to 30 in steps of 2 . The results for excitation pressures of $1 \mathrm{kPa}, 10 \mathrm{kPa}, 50 \mathrm{kPa}$ and $100 \mathrm{kPa}$ are presented in Figures 5-8.

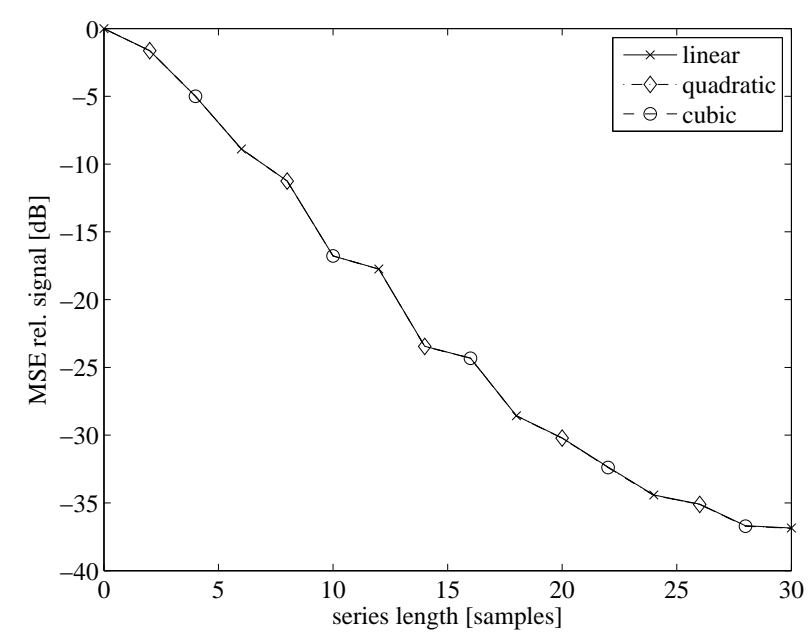

Fig. 5. Plot of MSE against filter length for $1 \mathrm{kPa}$ excitation pressure. The achievable MSE decreases with increasing filter length and attains a minimum value of $-36.9 \mathrm{~dB}$ at a series length of 30 samples. No difference in MSE may be attained by considering quadratic and cubic nonlinearities.

For an excitation pressure of $1 \mathrm{kPa}$, no difference in the mean-square error for linear, quadratic and cubic Volterra series can be seen. It can be thus concluded that at this excitation pressure, nonlinear effects may be neglected. At

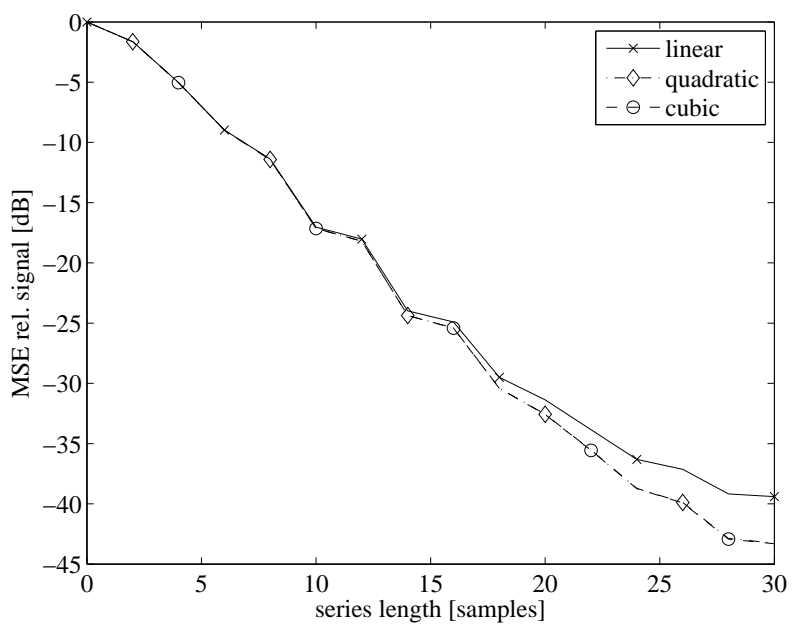

Fig. 6. Plot of MSE against filter length for $10 \mathrm{kPa}$ excitation pressure. A mean-square-error of $-39.4 \mathrm{~dB}$ is be attained for a linear model at a series length of 30 samples. The addition of quadratic terms improves the MSE by $3.9 \mathrm{~dB}$ to $-43.3 \mathrm{~dB}$.

higher insonification pressures, however, the nonlinearity of the microbubble oscillation becomes apparent. This can be seen in the plot of the MSE for an excitation pressure of $10 \mathrm{kPa}$. At a series length of 30 samples, an addition of the quadratic component yields an improvement of the meansquare error of $3.9 \mathrm{~dB}$ versus a linear model. The addition of cubic components does not yield an improvement in MSE.

These components are increasingly important at insonification pressures higher than $50 \mathrm{kPa}$. For this pressure, with a series length of 30 sample, an improvement of the 


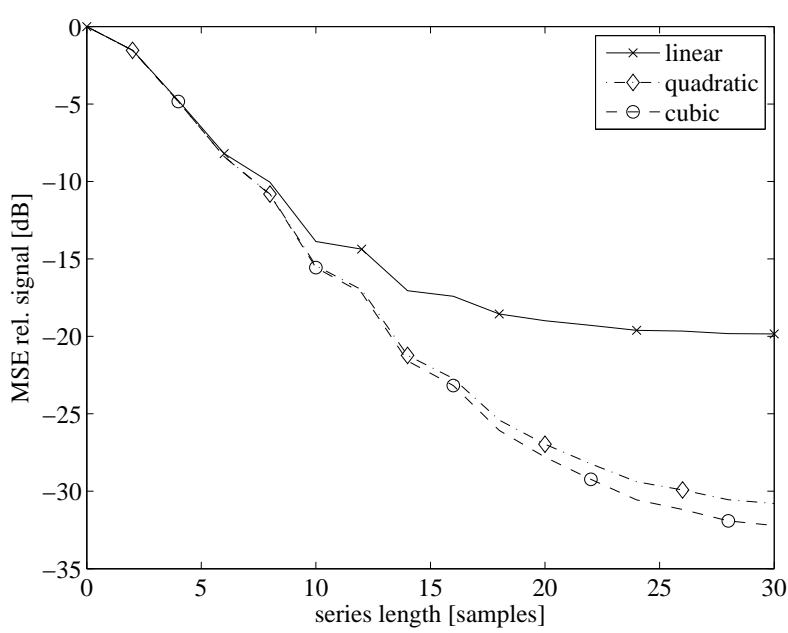

Fig. 7. Plot of MSE against filter length for $50 \mathrm{kPa}$ excitation pressure. The influence of nonlinear effects on bubble oscillation increases. For a linear model, the best achievable MSE is $-19.8 \mathrm{~dB}$. The addition of quadratic and cubic terms decreases this error to values of $-30.8 \mathrm{~dB}$ and $-32.2 \mathrm{~dB}$ respectively.

MSE of $1.4 \mathrm{~dB}$ can be accomplished by adding cubic terms to the identified Volterra series. The improvement gained

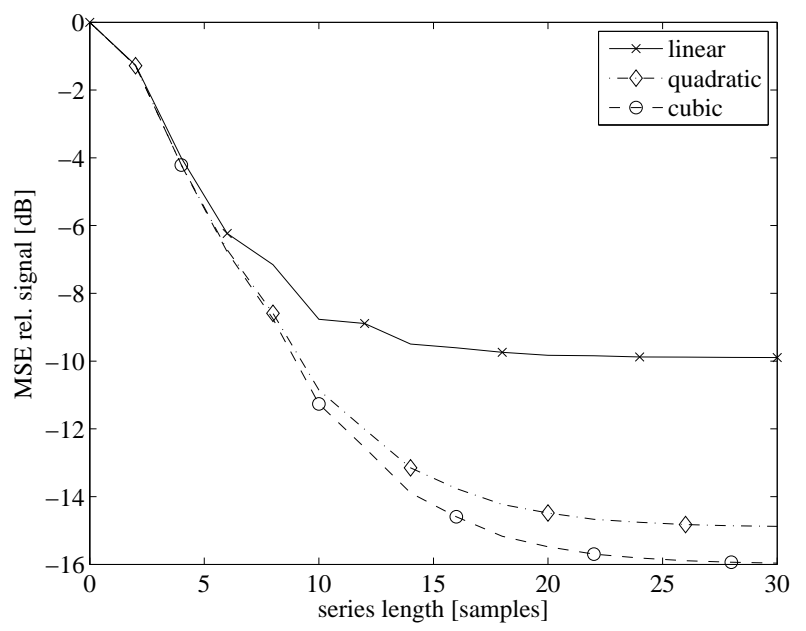

Fig. 8. Plot of MSE against filter length for $100 \mathrm{kPa}$ excitation pressure. The dominance of nonlinear effects increases further. A linear model only enables estimation with a minimum MSE of -9.9 dB. A Quadratic model improves the attainable MSE to $-14.9 \mathrm{~dB}$ and the addition of cubic terms reduces the MSE to $-16.0 \mathrm{~dB}$.

by adding cubic terms to the series increases with excitation pressure relative to the total mean-square error. For $100 \mathrm{kPa}$, the addition of cubic terms improves the mean square error by $1.1 \mathrm{~dB}$ to a total of $-16 \mathrm{~dB}$.

\subsection{Bubble motion for a narrowband excitation}

It can be seen, however, that the total achievable meansquare error increases with excitation pressure. This is due to the fact that the maximum order of the Volterra series used was a cubic model. Thus, at most third order effects are taken into account. The impact of this limitation may be best evaluated by considering the response of a microbubble to a narrowband excitation signal. For this, a modulated Gaussian envelope pulse was used. The center frequency of the pulse was chosen at $f_{0}=2.5 \mathrm{MHz}$ and the fractional bandwidth was chosen to be $B=8 \%$. The responses of the RPNNP and Volterra models to the pulse are shown in Figure 9. It can be seen that, although the general form of the curve is similar, significant differences can be seen at the peaks. The reason for this becomes apparent considering the frequency spectrum of the scattered pressure, shown in Figure 10.

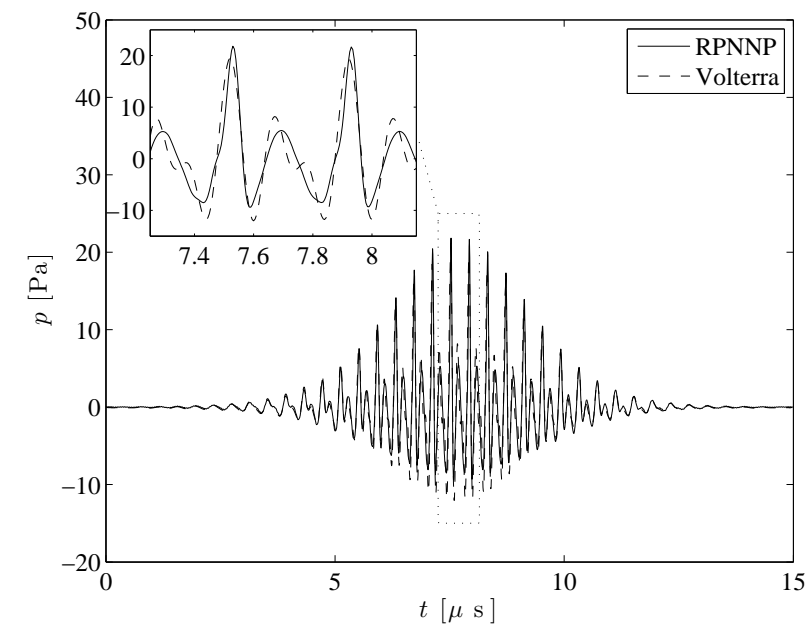

Fig. 9. Response of RPNNP (solid line) and Volterra series (dashed line) to a modulated Gauss envelope pulse, peak amplitude $100 \mathrm{kPa}$, center frequency $f_{0}=2.5 \mathrm{MHz}$, fractional bandwidth $B=8 \%$.

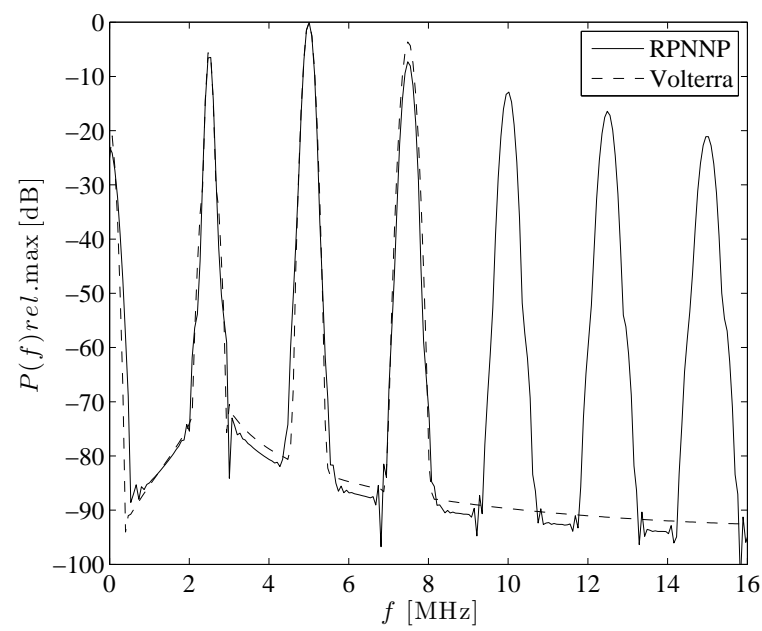

Fig. 10. Spectral response of RPNNP and Volterra series models to modulated Gaussian pulse. It can be seen that the estimation by the Volterra series corresponds very well to the RPNNP model up to the third harmonic. Higher harmonics than the third cannot be modeled since the cubic Volterra model used for estimation can at most produce third harmonics. 
While the fundamental as well as the second and third harmonics are represented with very high accuracy, higher harmonics are not considered at all. This is due to the nature of a third order Volterra series which may only create up to third harmonic components. This limitation may be circumvented by identifying also fourth order terms. Transducers usually used for signal transmission and reception, however, are inherently band-limited. Furthermore, sound attenuation in tissue increases exponentially with frequency. Therefore, effects attributed to higher orders than the third can often be disregarded in practice. To prove this point, the response of the RPNNP-equation as well as the Volterra series is shown after filtering with the impulse response of a typical transducer used in diagnostic imaging. The transducer is characterized by a center frequency of $f_{0 t}=3.5 \mathrm{MHz}$ and a fractional bandwidth $B_{t}=65 \%$. Figures 11 and 12 show the time domain and frequency domain responses of the RPNNP as well as the Volterra series models.

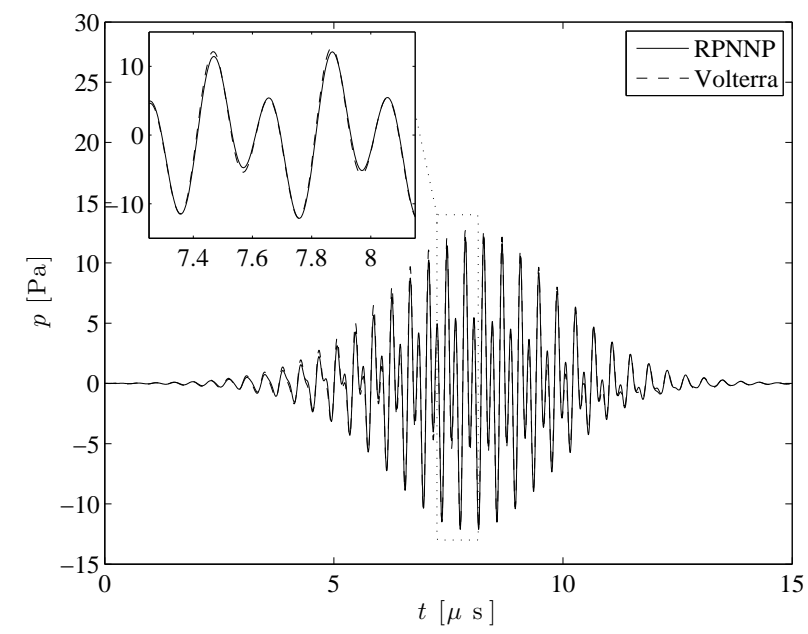

Fig. 11. Response of RPNNP (solid line) and Volterra series (dashed line) to a modulated Gauss envelope pulse, peak amplitude $100 \mathrm{kPa}$, center frequency $f_{0}=2.5 \mathrm{MHz}$, fractional bandwidth $B=8 \%$. Response was filtered with a transducer impulse response, center frequency $f_{0 t}=3.5 \mathrm{MHz}$, fractional bandwidth $B_{t}=65 \%$.

It can be seen that in the time domain, both models show very good correspondence. This is confirmed by the frequency domain data, with the signal level of the fourth and higher order harmonics being more than $60 \mathrm{~dB}$ below the maximum of the spectrum. Thus, for the bubble model evaluated within the scope of this paper, at sound pressure levels up to values of $100 \mathrm{kPa}$, the Volterra model provides a reasonably good fit to the system movement as determined by the reference model. To improve the performance at higher insonification pressures, the Volterra series model could be extended with higher order terms at the expense of increasing the complexity of the identification process. Higher pressures, however, also increase the probability of destruction of the microbubble by dissolution or inertial cavitation. Exams are usually conducted at insonification

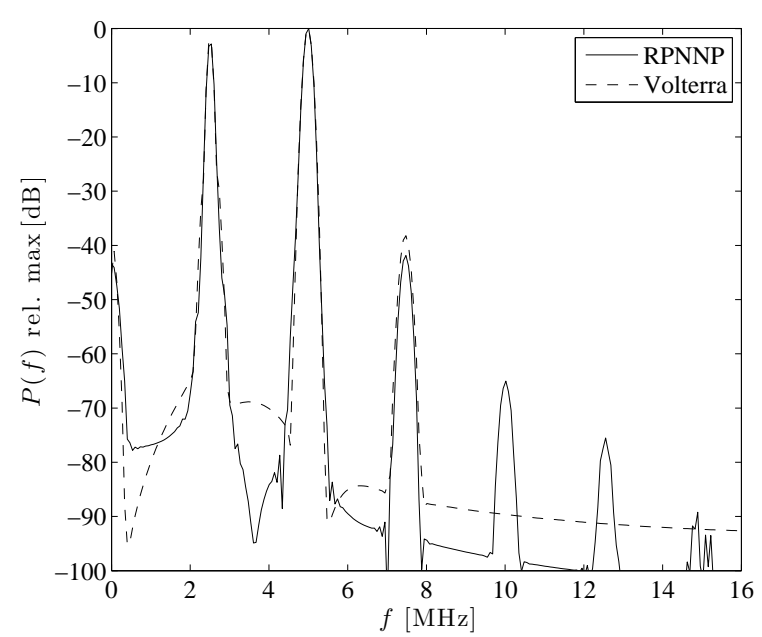

Fig. 12. Spectral response of RPNNP and Volterra series models to modulated Gaussian pulse, filtered by transducer impulse response. Due to the limiting of the bandwith by the ultrasound transducer, high order harmonics are suppressed. A third order model is thus suitable for the modeling of the oscillation characteristics of a microbubbles at this pressure.

pressures below this range. Thus, because of the band limitation of ultrasound transducers and the limited durability of microbubbles under high pressure insonification, the quality of the fit attained by the Volterra can be considered sufficient.

\subsection{Limitations of proposed model}

Additionally to the drawbacks outlined earlier, a limitation is that it is not possible to model subharmonics with a Volterra series model. This can be easily seen from (1). For a sinusoidal input, only integer multiples of the insonification frequency may appear in the output. Mitigations to this problem exist [14], however, and may be used to enable the modeling of subharmonics.

\section{Conclusions and Outlook}

It was shown that for moderate insonification pressures a Volterra series enables an adequate representation of the oscillation behavior of ultrasound contrast agents. For the model evaluated within the scope of this paper, accuracy was satisfactory up to insonification pressures of up to 100 $\mathrm{kPa}$. For practical purposes, however, this limitation can be disregarded, since the reception of harmonics of high order is hampered by the limited bandwidth of ultrasound transducers and the probability of microbubble destruction through dissolution or inertial cavitation increases with insonification pressure.

In conclusion, although the model presented does not allow for the modeling of subharmonics and increases in complexity with increasing insonification pressures, the form of description of the oscillation behavior may benefit the de- 
velopment of novel detection schemes for ultrasound contrast agents. Furthermore, a system identification approach enables the determination of nonlinear model from acoustic measurements, thus enabling the experimental quantification of the nonlinear parameters of ultrasound contrast agent microbubbles.

\section{References}

[1] N. de Jong, R. Cornet, C. T. Lancee, Higher harmonics of vibrating gas-filled microspheres. I: Simulations, Ultrasonics 32 (6) (1994) 447-453.

[2] N. de Jong, R. Cornet, C. T. Lancee, Higher harmonics of vibrating gas-filled microspheres. II: Measurements, Ultrasonics 32 (6) (1994) 455-459.

[3] D. H. Simpson, C. T. Chin, P. N. Burns, Pulse inversion doppler: A new method for detecting nonlinear echoes from microbubble contrast agents, IEEE T Ultrason Ferr 46 (6) (1999) 372-382.

[4] P. J. Phillips, Contrast pulse sequences (CPS): imaging nonlinear microbubbles, Proc IEEE Int Ultrason Symp (2001) 1739-1745.

[5] Lord Rayleigh, On the pressure development in a liquid during the collapse of a spherical cavity, Philos Mag 32 (8) (1917) 94-98.

[6] P. J. Frinking, N. de Jong, Acoustic modeling of shellencapsulated gas bubbles., Ultrasound Med Biol 24 (4) (1998) 523-533.

[7] K. E. Morgan, J. S. Allen, P. A. Dayton, J. E. Chomas, A. L. Klibaov, K. W. Ferrara, Experimental and theoretical evaluation of microbubble behavior: effect of transmitted phase and bubble size, IEEE T Ultrason Ferr 47 (6) (2000) 1494-1509.

[8] V. Volterra, Theory of functionals and of Integral and Integrodifferential equations, Dover Publishing, New York, 1930.

[9] M. Schetzen, The Volterra and Wiener Theories of Nonlinear Systems, John Wiley and Sons, New York, 1980.

[10] M. J. Korenberg, A robust orthogonal algorithm for system identification and time-series analysis, Biol Cybern 60 (1989) 267-276.

[11] R. D. Nowak, B. D. van Veen, Random and pseudorandom inputs for Volterra filter idenification, IEEE T Sig Proc 42 (1994) 21242135.

[12] M. Postema, G. Schmitz, Bubble dynamics involved in ultrasonic imaging, Exp Rev Mol Diag 6 (3) (2006) 493-502.

[13] L. D. Landau, E. M. Lifshitz, Fluid Mechanics, 2nd Edition, Pergamon Press, Oxford, 1987.

[14] O. M. Boaghe, S. A. Billings, Subharmonic oscillation modeling and MISO Volterra series, IEEE T Circuits-I 50 (7) (2003) 877884 